\section{DIFFUSE PHLEBOSCLEROSIS WITH CALCIFICATION}

BY

\section{A. Charan, M.S.}

Lecturer in Surgery, K.G. Medical College, Lucknow

An unusual case of diffuse phlebosclerosis with calcification is presented. No similar case has been found in the literature.

\section{Case History}

A Hindu male aged 20 was admitted on March 9, 1951, with increased frequency of micturition, dysuria, and haematuria of two days' duration. He also complained of lumps in front of the elbows and behind the knees, which had apparently first been noticed by his parents soon after his birth and had since been slowly increasing in size. He had had dysentery during childhood and two recent attacks of asthma. He had also had two severe attacks of renal colic

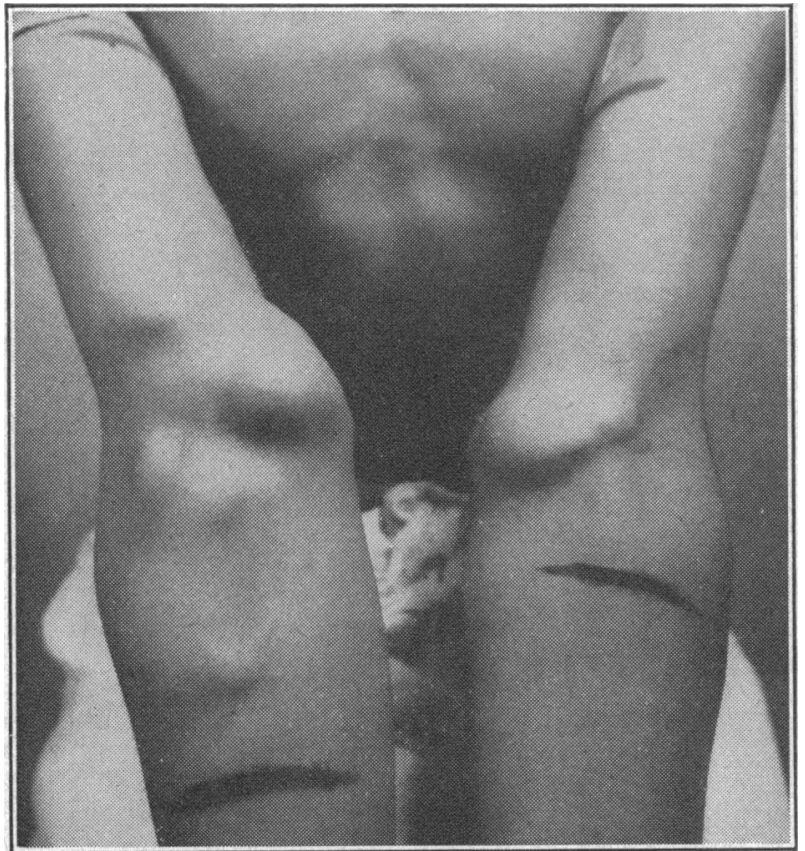

FIG. 1.-Photograph showing both arms and forearms with bonyhard cord-like swellings. Their extent is marked.

four years and two and a half years previously, lasting several hours each; haematuria had not been present on these occasions. There was no relevant family history.

On examination the patient was shocked and restless with a pulse rate of 120 . There were bilateral, bony-hard, nodular, cord-like swellings in the lower two-thirds of the arms, extending across the cubital fossa into the anterior aspect of the upper half of the forearm (Fig. 1). The swellings were fixed to the deeper structures, and related to the main vascular bundle of the limb. The brachial and radial pulses were very feeble on both sides, but the arterial walls were not palpably thickened. The axillary pulse was normal. Clinically the swellings appeared to be calcified venous varices. Similar swellings were present bilaterally in the legs, extending from the lower third of the thigh, across the popliteal fossa, to half-way down the back of the calf. The pulsation of the popliteal and posterior tibial arteries was greatly reduced on both sides, but that of the femoral artery was normal. There were no signs of peripheral vascular insufficiency. No other physical abnormalities were discovered except a urethral stricture $1 \frac{1}{2}$ in. $(3.8 \mathrm{~cm}$.) proximal to the urinary meatus.
Investigations.-Urine, S.G. 1020 ; albumin, + ; large numbers of red blood cells ; sterile on two occasions. Renal function: non-protein nitrogen, $50 \mathrm{mg}$. per $100 \mathrm{ml}$.; blood urea, $43 \mathrm{mg}$. per $100 \mathrm{ml}$.; urea concentration, $2.2 \%$. Blood : white cells, 15,650 per c.mm. (poly. $83 \%$, lymph. $9 \%$, eosin. $2 \%$, mono. $6 \%$ ) ; red cells, $3,380,000$ per c.mm.; $\mathrm{Hb}, 54 \%$ (8.1 g.); slight anisocytosis and moderate vacuolation also present; bleeding-time, 2 minutes 55 seconds; coagulation time, 5 minutes 15 seconds (both within normal limits); serum calcium, $10.2 \mathrm{mg}$. per $100 \mathrm{ml}$. ; serum phosphorus, $4 \mathrm{mg}$. per $100 \mathrm{ml}$; alkaline phosphatase, 6 units (similar results on two occasions); Sulkowitch's test, no increase of urinary calcium excretion; Kahn test and Wassermann reaction negative.

Radiological examination.-Plain $x$-ray pictures of the arms (Fig. 2), legs (Fig. 3), head and neck, and renal pedicle

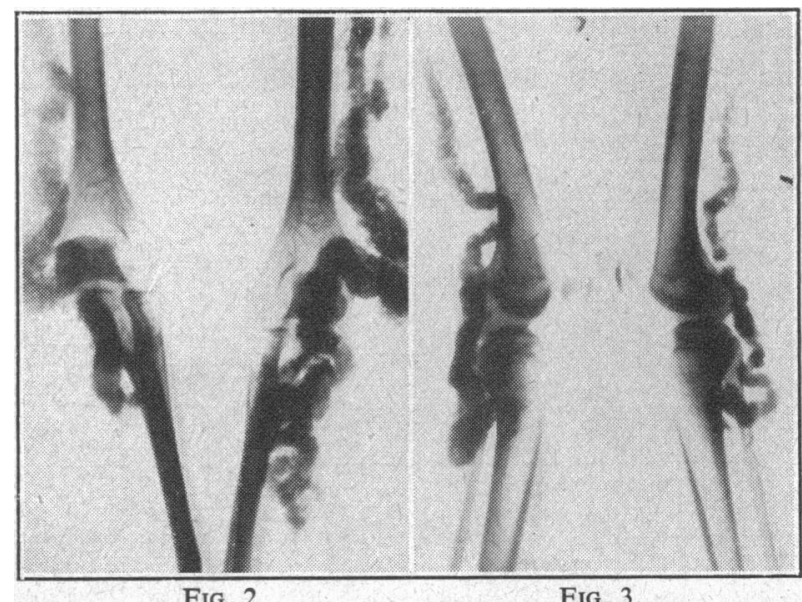

Fig. 2.-Radiograph of arms, showing radio-opaque cord-like shadows of calcified veins. FIG. 3.-Radiograph of legs showing radio-opaque cord-like shadows of calcified veins.

showed calcified cord-like shadows, provisionally diagnosed as calcified veins. Descending pyelography showed both kidneys ; the left pelvis and upper part of the ureter were not so well seen as on the right; the dye reached the bladder.

Treatment and Operative Investigations.-For the haematuria he was given three blood transfusions (totalling $1,050 \mathrm{ml}$.), bladder irrigations, and coagulants such as calcium, "coagulin," and vitamin $K$. The swelling in the left arm was excised (Fig. 4). It was a calcified tortuous mass, replacing the brachial vein and adherent to the brachial artery and median nerve. The brachial artery and the other venae comitantes were normal. The cut surface of the specimen was yellowish white, granular, and gritty to the touch. Histological examination showed a circular mass of fibrous tissue in which were several foci of calcium deposit, each surrounded by dense collagenous tissue. In the fibrous bands between the calcium deposits there were small capillaries. No elastic or muscular tissue was seen. The parathyroid glands were explored, but were macroscopically normal. Pyelolithotomy was undertaken on the right side, but, apart from the presence of a calculus and calcified renal veins, no other abnormality was discovered.

\section{Discussion}

The clinical nature of the swellings, together with $x$-ray and histological

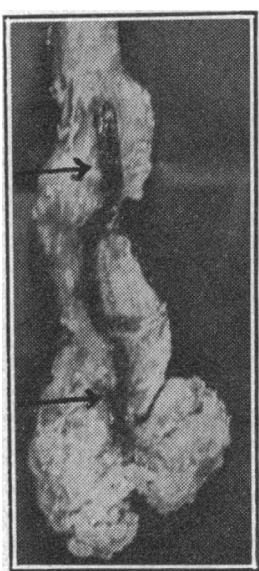

Fig. 4.-Specimen of the cord-like hard tortuous vein removed at operation from the left arm The arrow shows the normal vena comitans. 
findings, leaves little doubt that they were venous varices, whose lumen had become obliterated and undergone secondary calcification.

The differential diagnosis comprised parathyroid dyscrasia with disturbed calcium metabolism; other causes of pathological calcification ; and primary degenerative vascular conditions with secondary calcification. Parathyroid dyscrasia is ruled out by the negative clinical, biochemical, radiological, and surgical findings. Phlebolith, calcified parasites, myositis ossificans, etc., can be excluded on the physical character of the lumps, the $x$-ray findings, and the absence of their respective signs and symptoms. Of the degenerative vascular conditions, arteriosclerosis and Mönckeberg's degeneration can be excluded in view of the age of the patient and the absence of evidence of any organic changes in the arteries.

A diagnosis of phlebosclerosis is thus very appropriate, and most of the features of this case can be explained by it.

A review of the literature on phlebosclerosis shows that reports of the condition are uncommon. Before 1905 there is hardly any reference to it, and it had been described as occurring in elderly persons and associated with patchy subintimal fibrosis, calcification being unknown. Martin and Meakins (1905), Stahl and Zeh (1923), and Hauswirth and Eisenberg (1931) defined the condition more closely, calling it venofibrosis, peripheral phlebosclerosis, hyperplastic endophlebitis, etc. They describe it as of slow and gradual course and that it is seen in patients between 18 and 45 years of age. It has been associated with cases of gout, lead-poisoning, and syphilis. Hauswirth and Eisenberg found a high incidence of the condition in peptic ulcer cases, but did not encounter oedema, necrosis, or calcification. Histologically they found hyalinization of the intima and hyperplasia of fibrous tissue in the media, leading to obliteration of the lumen. It was progressive and painless. Boyd (1947) has described phlebosclerosis as occurring between the ages of 20 and 30, unassociated with arteriosclerosis, vascular insufficiency, or inflammation. Fatty degeneration or calcification is always bilateral, and affects both superficial and deep veins, which feel like hard mobile cords. The lumen is narrowed and there is medial fibrosis. Levin and Bucy (1936) have described a case of proliferative endophlebitis with phlebosclerosis : superficial veins were firm, small, cord-like, and not varicose.

In the present case the swellings were first noticed soon after birth, and there is therefore a strong suspicion of their being congenital. Their pathogenesis can be explained only on the basis of some abnormal developmental process. Possible hypotheses are : (1) that calcification has occurred in areas of abnormal vascular formation where canalization has not taken place ; or (2) at a later stage, after vein formation, ischaemia has occurred because of an inadequate blood supply from the vasa vasorum, with subsequent calcification. The presence of renal calculus is difficult to explain. and may be coincidental.

I am indebted to Professor S. N. Mathur, head of the department of surgery and superintendent, Gandhi Memorial and Associated Hospitals, for permission to publish the case, and to Professor S. C. Misra, professor of clinical surgery, Professor V. S. Manglik, professor of pathology, and to other colleagues for their help and advice.

\section{References}

Boyd, W. (1947). Textbook of Pathology. London.

Hauswirtb L., and Eisenberg. A. A. (1931). Arch. Path., 11, 857 Levin, P. M.. and Bucy, P. C. (1936). Arch. intern. Med., 57, 787 Martin, C F., and Meakins, J. C. (1905). Amer. Med., 10, 611 Stahl, R.. and Zeh, F. (1923). Virchows Arch. path. Anat., 242, 70.

The tendency for certain organizations to raise hopes of adoption before the birth of an illegitimate child is condemned in the recently issued annual report of the National Council for the Unmarried Mother and her Child. This sometimes leads to inadequate antenatal preparations on the part of the mother, since she feels certain that she will give up her child on leaving hospital. However, once the baby has arrived she often changes her mind.

\section{Medical Memorandum}

\section{Neonatal Death due to Ligation of Umbilical Cord by an Amniotic Band}

The following case of neonatal death is interesting enough to be placed on record. So far as we can ascertain no previous foetal death due to this exact cause has been published.

\section{CASE REPORT}

The patient was a healthy multipara aged 26 , with one previous live baby. Her first confinement, four years previously, was conducted by another practitioner, and was normal in every respect.

Her second pregnancy was associated with much sickness in the early months. The presentation in the early part of the eighth month was a breech, which underwent spontaneous version. The final presentation, confirmed by $x$ ray examination, was a normal vertex. The expected date of delivery was about March 28, 1951. There was no clinical evidence of hydramnios, and the case was regarded as a normal pregnancy.

F oet a 1 movements were vigorous up to the morning of March 17 , when they suddenly stop ped. Unfortunately an op portunit y of examining the patient at this stage did not arise, and she went into labour in the early

hours of March 18. Labour proceeded rapidly, and the passage of meconium was noted by the nurse when the membranes ruptured at 4.50 a.m. One of us was summoned by the nurse, and on arrival about 30 minutes later a dead baby had just been delivered. So far as we can judge, the first stage took about three hours and the second stage about three-quarters of an hour.

The appearance was very remarkable. The placenta had not yet been delivered, and issuing from the vagina was a thin cord-like structure which was knotted tightly round the umbilical cord about 5 in. $(12.5 \mathrm{~cm}$.) from the umbilicus. The baby, a male of $6 \mathrm{lb} .10 \mathrm{oz}$. $(3 \mathrm{~kg}$.) was not macerated. and appeared normal. The nurse had cut the cord proximal and distal to the constricting band. The placenta was delivered after a normal period without any difficulty.

Examination of the placenta and the cord-like band showed that the latter arose from the amnion and that its length from the edge of the placenta to the point of constriction on the cord was $22 \mathrm{in}$. $(56 \mathrm{~cm}$.). Its total length was 31 in. $(78.5 \mathrm{~cm}$.). The appearance is well demonstrated in the accompanying photograph.

A possible explanation of the mechanism of the constriction is that the umbilical cord had been encircled by the amniotic band in a loose knot during spontaneous version, and that the constriction was fatally tightened with the descent of the head into the pelvis about or immediately before the onset of labour. This would seem to be borne out by the fact that the foetal movements suddenly ceased about 15 hours before the onset of labour.

R. O'K. Craven, M.D.

C. J. M. Gadoes, M.B., Ch.B. 